\title{
Controlling of mechanical property in additive manufactured porous titanium by structural control and alloying for bone substitutes
}

\author{
Masato Ueda ${ }^{\mathrm{a}, *}$, Masahiko Ikeda ${ }^{\mathrm{a}}$ \\ ${ }^{\text {a }}$ Department of Chemistry and Materials Engineering, Faculty of Chemistry, Materials and Bioengineering, Kansai University \\ *m-ueda@kansai-u.ac.jp
}

$\underline{\text { Abstract }}$

Mechanical properties of metallic materials can be controlled by not only alloy design but also constructing appropriate structure. A porous material with adequate pore structure showing appropriate mechanical properties has long been sought as the ideal bone substitute, because it exhibits low Young's modulus and bone ingrowth. Additive manufacturing (AM) can produce metallic tailor-made products such as artificial bone, several joints etc. The purpose of this work was to control the mechanical property of porous Ti by controlling the porous structure. In addition, the characteristics of Ti-Zr-Fe alloys were also investigated as the materials for the AM. First, porous polylactic acid with rhombicuboctahedron-derived structure was prepared by a 3D printer to determine appropriated structure for bone substitutes. The compressive strength and Young's modulus was strongly influenced by the minimum cross-sectional area fraction perpendicular to the loading direction. Then the porous Ti with similar structures were prepared by a laser AM. The strength and Young's modulus were extremely low compared with the expected ones. Then Ti-xmass $\% \mathrm{Zr}-1$ mass $\% \mathrm{Fe}$ alloys $(x=0,5,10)$ were prepared as the materials for the AM. Vickers hardness increased almost linearly with $\mathrm{Zr}$ content by solution hardening. Ideal bone substitutes would be produced by such structural design and alloying.

\section{Introduction}

Titanium and it alloys have been used widely as biomaterials for orthopaedic implants because of their excellent mechanical properties, excellent corrosion resistance and biocompatibility. However, such metallic materials often cause stress shielding due to mismatch of Young's modulus between the implants and the surrounding bone. For example, the Young's moduli of pure Ti and cortical bone are 110 and 10-30 GPa, respectively. As a result of this mismatch, the bone around such metallic implants is insufficiently loaded, thus causing bone atrophy [1]. Eventually the implants loosen clinical performance.

Mechanical properties of metallic materials can be controlled by not only alloy design but also constructing appropriate structure [2-5]. A porous material with adequate pore structure showing appropriate mechanical properties has long been sought as the ideal bone substitute, because it exhibits low Young's modulus and bone ingrowth.

Recently, great advancements have been made in additive manufacturing (AM) technology, also commonly known as three-dimensional (3D) printing. Powder bed fusion is a common technology used in 3D printing, particularly when applied to metallic materials. The layer-by layer build-up technique, can produce metallic tailor-made products such as artificial bone, several joints etc [6-8].

Going back to the purpose of this study, it must be also important for controlling mechanical property of metallic devices to prepare dedicated powders designed for the AM in addition to the structural design. Up to now, various Ti alloys such as Ti-V-, Ti-Mo- systems have been developed for several applications. The element of zirconium (Zr) belongs to the same group 4 as Ti in the periodic table. Therefore it possesses similar chemical properties. Recently, pure $\mathrm{Zr}$ or $\mathrm{Zr}$ based alloys have received much attention in the field of biomaterials comparable to $\mathrm{Ti}$ based alloys [9,10], because they do not directly bond to living bone [11] or show low magnetic susceptibility [12]. Furthermore, the Ti-Zr binary system forms a continuous solid solution for both high temperature $\beta$ phase with the body centered cubic (BCC) structure and low temperature $\alpha$ phase with the hexagonal closed packed (HCP) structure throughout the entire range of composition [13]. As is well known, on the other hand, the element of iron (Fe) is not only inevitable but also effective element in Ti [14]. By incorporating Fe at the stage of alloy design, off-grade sponge titanium can be employed. Both elements seem to be effective in strengthening the titanium alloys.

The purpose of this work was to control the mechanical property of porous Ti by controlling the porous structure. In addition, the characteristics of Ti-Zr-Fe alloys were also investigated as the materials for the AM.

\section{Material and experiments}

2.1 Structural design of porous devices

The computer-aided design (CAD) of the porous structure was conducted using Shade 3D professional $\quad$ 15.0.1 (Shade 3D Co., Ltd.). Porous polylactic acid (PLLA) with rhombicuboctahedron-derived structure (Fig.1) was prepared by a conventional 3D printer (Value 3D MagiX: MF-1000). The entire porous structure consisted of 180 units $(6 \times 6 \times 5$ in the width, in-plane depth, and height directions) and the size was almost $24 \times 24 \times 42(\mathrm{~mm})$. In the present study, the parameter of $W / l$, that is, the neck of a connection part of the unit structures was focused.

A pure Ti powder (ASTM grade 2, average particle size $<23 \times 10^{-6} \mathrm{~m}$ ) was prepared by gas atomisation. Porous Ti with the rhombicuboctahedron-derived structure were also fabricated with the EOSINT M 270 laser AM machine (EOS, Germany), which has a $200 \mathrm{~W}$ fibre laser with high beam quality good power stability. In these samples, the entire porous structure consisted of 216 units $(6 \times 6 \times 6$ in the width, in-plane depth, and height directions) and the size was almost $6 \times 6 \times 12(\mathrm{~mm})$.

(C) The Authors, published by EDP Sciences. This is an open access article distributed under the terms of the Creative Commons Attribution License 4.0 (http://creativecommons.org/licenses/by/4.0/). 
The fabricated prismatic samples were used in a mechanical test. A compression test was conducted for the porous samples using a tensile tester (Autograph AG-X Plus, Shimadzu, Japan) with a $5 \mathrm{kN}$ load cell. The initial strain rates were selected to be $1.67 \times 10^{-4} \mathrm{~s}^{-1}$ and $1.74 \times 10^{-3} \mathrm{~s}^{-1}$ for porous PLLA and porous Ti, respectively. A mechanical test using a strain gauge is necessary to determine Young' modulus of samples. However it could not be applied to the present porous samples. In the present study, the strain was obtained from the stroke displacement and the gradient of the stress-strain curve in elastic region was defined as apparatus Young's modulus. The $0.2 \%$ proof stress of the samples was also determined from the stress-strain curves.

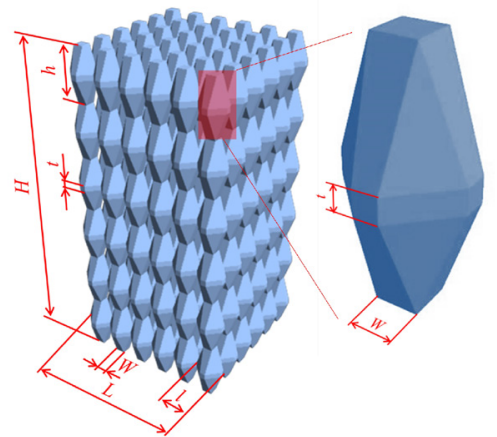

Fig.1 Rhombicuboctahedron-derived structure

Ti-xmass $\% \mathrm{Zr}-1 \mathrm{mass} \% \mathrm{Fe}$ alloys $(x=0,5,10)$ were melted in a laboratory-scale arc furnace under a high purity argon atmosphere from the sponge Ti $(>99.8 \%$, OSAKA Titanium technologies Co., Ltd.), the sponge $\mathrm{Zr}$ (99.9\%, Mitsuwa Chemicals Co., Ltd.) and the Fe wire (99.5\%, The Nilako Corporation). The resulting button ingots were hot forged and rolled at approximately $1120 \mathrm{~K}$ to obtain plates of approximately $2 \mathrm{~mm}$ in thickness. The compositions of each of the Ti-Zr-Fe alloys were determined by X-ray fluorescence measurement. The phase constitutions were identified by X-ray diffraction (XRD) at room temperature. In the measurement, Si powder was put on the samples as a standard in order to correct the diffraction angle. The Vickers hardness (HV) of each sample was also measured at room temperature, using a load of $4.9 \mathrm{~N}$.

\section{Results and discussion}

3.1 Structural design of porous devices

Six types of porous PLLA samples were prepared by using the 3D printer. The fabricated porous structures appear as designed, though the horizontal streaks caused by the printed layers could be slightly observed. Then the compressive test was performed to compare the mechanical properties of the structures with different unit dimensions. All stress-strain curves were typical and smooth. The apparent Young's modulus and the proof stress were obtained from the curves. As expected, such mechanical properties were strongly influenced by the minimum cross-sectional area fraction perpendicular to the loading direction. The apparent Young's modulus was increased with increasing minimum cross-sectional area fractions, as shown in Fig.2. This result indicates the Young's modulus of porous devices can be easily and effectively controlled by adjusting the structural parameters of the present structure.

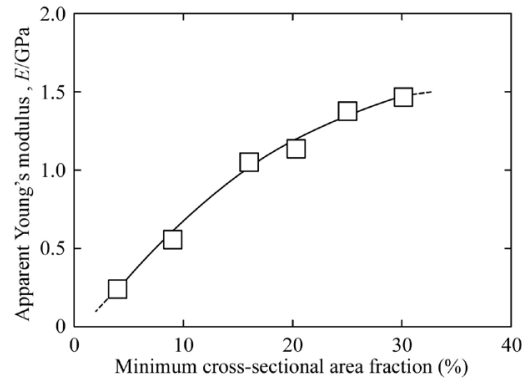


Appropriate structure could be determined from the preliminary experiments of porous PLLA and then five types of porous structures were produced in pure Ti by the laser AM. The fabricated porous structures appear as designed, though the flat planes in the rhombicuboctahedron-derived unit structure could not be precisely reproduced as a result of surface tension during melting. This is not a problem at all. The connection region in the longitudinal direction was observed by scanning electron microscopy (SEM) as shown in Fig. 3. The shape of the powders remained on the surface in all samples. The structural shape of the connection region could not be accurately reproduced that in the CAD designed in some cases. For the porous Ti with small $W / l$ values, the neck of the connection region was confirmed to show a cylindrical shape. Interestingly, the powders were completely melted in the connection regions. This may have been due to intensive heat flow induced by the structure. The heat flow from the thick parts seemed to be hindered by the thin necks. It means that the structural design that takes into account the heat flow and solidification speed etc. is necessary.

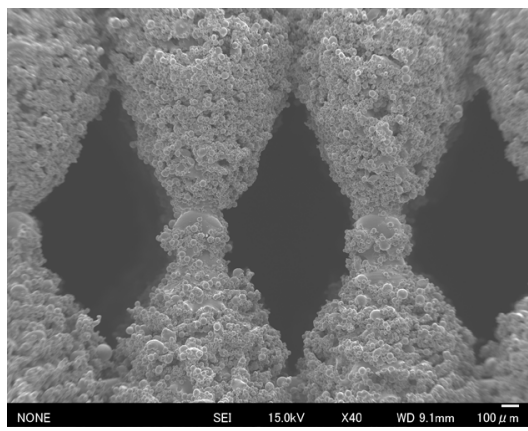

Fig.3 SEM image of the neck of a connection region in additively manufactured porous Ti sample with $W / l=0.35$

Compressive tests were performed to compare the mechanical properties of the structures with different W/l values. All stress-strain curves were typical and smooth, as in ductile bulk metals. The apparent Young's modulus and the proof stress were obtained from the curves and were strongly influenced by the W/1 values as shown in Fig.4. The apparent Young's modulus gradually increased with the W/1 values, and saturated above $W / l=0.8$, and decreased slightly in the dense sample prepared as a rectangular parallelepiped. The yield stress increased drastically with increasing $W / l$. The yield stress could not be measured in the porous Ti samples with $w / l=0.8$ and 0.95 and the dense sample because of the limitations of the load cell, $5 \mathrm{kN}$. The result indicates that the present structural control is effective in tuning of the mechanical properties of porous Ti as well. The Young's modulus is not real one because it is obtained from the stress-strain curve without strain gauge. However it might be low compared with the expected ones.

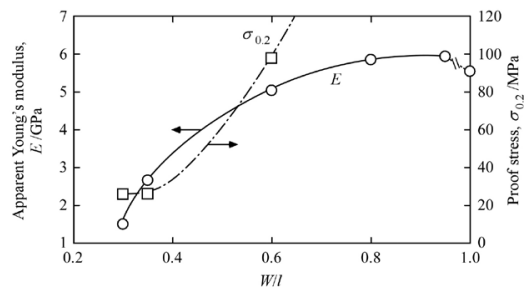

Fig.4 Apparent Young's modulus and $0.2 \%$ proof stress in porous Ti with different W/ values.

As mentioned above, the mechanical property of the AMed pure Ti might be slightly lower than that of melted one. It must be important for controlling mechanical property of porous materials to prepare dedicated powders designed for AM in addition to the above structural design. Then Ti- $x$ mass $\% \mathrm{Zr}-1$ mass $\% \mathrm{Fe}$ alloys $(x=0,5,10)$ were prepared as the materials for the AM. Well-mixed and homogeneous ingots could be obtained by the present method. The chemical compositions were determined to be Ti-0Zr-1.0Fe, Ti-4.7Zr- $0.9 \mathrm{Fe}$ and Ti-9.5Zr-1.0Fe for the alloys by X-ray fluorescence analysis, which were almost the same as the nominal ones. On the other hand, oxygen contaminations were less than $0.09 \%$. Figure 5 shows X-ray diffraction patterns of the arc-melted alloys. Obvious peaks attributed to $\alpha$ phase could be confirmed to appear in all alloys. Although the microstructures are not shown here, all samples show similar and typical microstructures of $\alpha$ phase. Figure 6 shows the Vickers hardness as a function of $\mathrm{Zr}$ content. Vickers hardness increased almost linearly with $\mathrm{Zr}$ content from 160 to 210 by solution hardening. The mechanical property might be improved nearly 1.3 times without changing microstructure and phase constitution. Therefore, ideal porous bone substitutes would be produced by the present alloy design by combining with the structural control described above. 


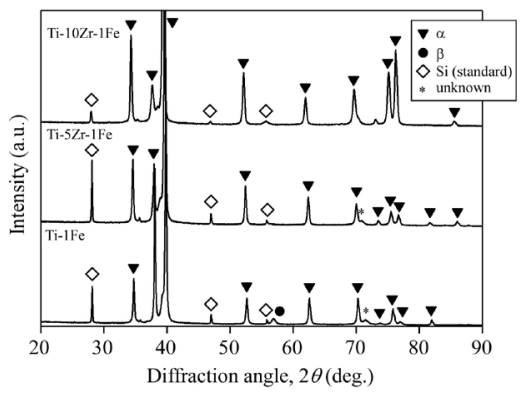

Fig.5 $\mathrm{X}$-ray diffraction patterns of $\mathrm{Ti}-1 \mathrm{~F}, \mathrm{Ti}-5 \mathrm{Zr}-1 \mathrm{Fe}$ and $\mathrm{Ti}-10 \mathrm{Zr}-1 \mathrm{Fe}$ alloys as arc-melted.

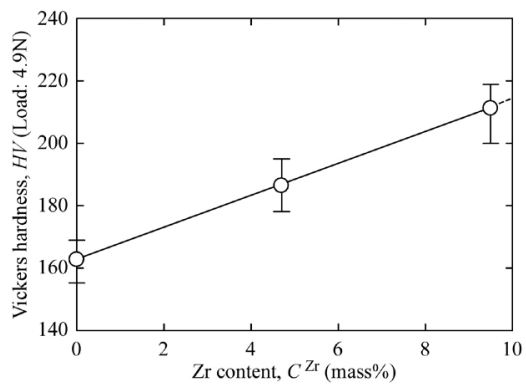

Fig.6 Change in Vickers hardness in Ti-Zr-Fe alloys depending on $\mathrm{Zr}$ content.

\section{Conclusion}

Porous Ti samples composed of rhombicuboctahedron-derived units were fabricated by laser additive manufacturing. The dependence of the mechanical properties of the fabricated samples on the structural parameters was investigated. The following conclusions can be drawn from this study. The characteristics of Ti-Zr-Fe alloys were also investigated as the materials for the additive manufacturing. The following conclusions can be drawn from this study.

1) Porous Ti structures composed of rhombicuboctahedron-derived units could be accurately fabricated as designed by laser additive manufacturing

2) The apparent Young's modulus increased as the minimum cross-sectional area ratio in the plane perpendicular to the loading axis increases. This indicates the mechanical property of porous Ti can be arbitrarily controlled by adjusting the design parameters of the present structure.

3) Vickers hardness increased almost linearly with $\mathrm{Zr}$ content from 160 to 210 by solution hardening in Ti-Zr-Fe alloys.

\section{Acknowledgements}

The authors would like to thank Chang Tinghsuan, Yohei Fujimoto and Maria Adachi for their assistance with the image analysis and the mechanical testing. This work was supported in part by the Light Metal Educational Foundation, Inc. 
[1] N. Sumitomo, K. Noritake, T. Hattori, K. Morikawa, S. Niwa, K. Sato, M. Niinomi, J. Mater. Sci. Mater. Med. 19 (2008) 1581-1586.

[2] T. Ahmed, M. Long, J. Silvestri, C. Ruiz and H.J. Rack, in Titanium'95: Science and Technology, P.A. Blenkinsop, W.J. Evans and H.M. Flower, eds., The Institute for Materials, Birmingham, UK (1996) pp. 1760-1767.

[3] D. Kuroda, M. Niinomi, M. Morinaga, Y. Kato, T. Yashiro, Mater. Sci. Eng. A 243(1-2) (1998) 244-249.

[4] N. Nomura, T. Kohama, I.H. Oh, S. Hanada, A. Chiba, M. Kanehira and K. Sasaki: Mater. Sci. Eng. C 25 (2005) 330-335.

[5] C.E. Wen, M. Mabuchi, Y. Yamada, K. Shimojima, Y. Chino, T. Asahina, Scripta Mater. 45 (2001) 1147-1153.

[6] B. Levine, Adv. Eng. Mater. 10 (2008) 788-792.

[7] G. Lewis, J. Mater. Sci. Mater. Med. 24 (2013) 2293-2325.

[8] R. Hedayati, S.A. Yavari, A.A. Zadpoor, Mater. Sci. Eng. C 76 (2017) 457-463.

[9] T. Hanawa, S. Hiromoto, K. Asami, O. Okuno, K. Asaoka, Mater. Trans. 43 (2002) 3000-3004.

[10] L. Saldaña, A. Me'ndez-Vilas, L. Jiang, M. Multigner, J. L. Gonza'lezCarrasco, M. T. Pe'rez-Prado, M. L. Gonza' lez-Martı'n, L. Munuera, N. Vilaboa, Biomaterials 28 (2007) 4343-4354.

[11] P. Thomsen, C. Larsson, L. E. Ericson, J. Mater. Sci.: Mater. In Medicine 8 (1997) 653-665.

[12] D. R. Ride, CRC Handbook of Chemistry and Physics, 87th edition, (CRC Press, Taylor \& Francis Group, (2006) pp. 4-142-4-147.

[13] J.L. Murray, Phase Diagrams of Binary Titanium Alloys, (ASM International, Metals Park, OH, 1987) pp. 340-345.

[14] S. Komatsu, M. Ikeda, T. Sugimoto, K. Kamei, K. Inoue, J.Japan Inst. Metals 55(1991) 491-496. 